\title{
Biomarkers in critical illness: have we made progress?
}

\author{
Patrick M Honore' \\ Rita Jacobs' \\ Inne Hendrickx' \\ Elisabeth De Waele' \\ Viola Van Gorp' \\ Olivier Joannes-Boyau ${ }^{2}$ \\ Jouke De Regt' \\ Willem Boer ${ }^{3}$ \\ Herbert D Spapen' \\ 'Intensive Care Unit, Universitair \\ Ziekenhuis Brussel, VUB University, \\ Brussels, Belgium; ${ }^{2}$ Intensive Care \\ Unit, Hopital Haut Leveque, University \\ of Bordeaux 2, Bordeaux, France; \\ ${ }^{3}$ Intensive Care Unit, Ziekenhuis \\ Oost-Limburg, Genk, Belgium
}

This article was published in the following Dove Press journal: International Journal of Nephrology and Renovascular Disease 17 October 2016

Number of times this article has been viewed

Abstract: Biomarkers have emerged as exemplary key players in translational medicine. Many have been assessed for timely recognition, early treatment, and adequate follow-up for a variety of pathologies. Biomarker sensitivity has improved considerably over the last years but specificity remains poor, in particular when two "marker-sensitive" conditions overlap in one patient. Biomarker research holds an enormous potential for diagnostic and prognostic purposes in postoperative and critically ill patients who present varying degrees of inflammation, infection, and concomitant (sub)acute organ dysfunction or failure. Despite a remarkable progress in development and testing, biomarkers are not yet ready for routine use at the bedside.

Keywords: biomarkers, acute kidney injury, sepsis, ARDS

\section{Biomarkers: protagonists of translational medicine}

Biomarkers hold considerable promise in current translational medicine. We previously discussed the role of biomarkers for early diagnosis of acute kidney injury (AKI) in critically ill patients. ${ }^{1}$ Meanwhile, other biomarkers have found the way from bench to bedside. Clinical experience has shown that some of these markers may become powerful tools in the management of critically ill patients. However, the complexity of acute disease conditions exhibits some pitfalls for biomarker use that need further investigation.

Renal biomarkers easily outperform "traditional" variables such as creatinine and estimated glomerular filtration rate to predict occurrence and outcome of AKI. ${ }^{1-3}$ However, many questions remain. Whether biomarkers can improve the approach to the sick or compromised kidney, in particular regarding timely initiation of continuous renal replacement therapy (CRRT), has insufficiently been clarified. Moreover, biomarkers lack specificity in case of sepsis-induced AKI or in AKI associated with the acute respiratory distress syndrome (ARDS). Also, a potential role of biomarkers to assist in decision processes for admission or discharge in emergency and intensive care units (ICUs) has not been established.

The present paper aims to briefly update actual knowledge on the (potential) use of biomarkers in critically ill patients.

Correspondence: Patrick M Honore Intensive Care Unit, Universitair Ziekenhuis Brussel, VUB University, 10I Laarbeeklaan, 1090 Brussels, Belgium $\mathrm{Tel}+3224749097$

Email Patrick.Honore@az.vub.ac.be

\section{Biomarkers and AKI: growing up from the cradle}

AKI is a major determinant of morbidity and mortality in critically ill patients. Crucial issues are early detection of the at-risk population and timely start of therapy. In this context, biomarkers may have a decisive preemptive role. 


\section{Neutrophil gelatinase-associated lipocalin (NGAL)}

Patients with predominant cardiorenal syndrome and high serum concentrations of NGAL were found to be at risk of developing AKI, even in the absence of oliguria and increased creatinine levels. NGAL-positive patients also more frequently require renal replacement therapy and have higher mortality rates..$^{2-4}$ This new entity, divulged by using a biomarker as "subclinical AKI", ${ }^{5}$ suggested that current concepts and definition of AKI might need reassessment. ${ }^{2}$

\section{Cycle cell arrest biomarkers}

The SAPPHIRE study ${ }^{6,7}$ showed that two novel cell cycle arrest biomarkers, tissue inhibitor of metalloproteinase-2 and urinary insulin-like growth factor binding protein 7, largely outperformed existing markers. Whether these markers can predict the need for or duration of renal replacement therapy is currently under investigation. Studies in ICU patients will help to identify the potential of tissue inhibitor of metalloproteinase-2/ insulin-like growth factor binding protein 7 for early detection of postoperative or antimicrobial-related toxic AKI.

\section{Cystatin C (Cys-C)}

High serum Cys-C levels at admission were found to predict subclinical AKI in patients admitted in the ICU. ${ }^{8}$

\section{Vitamin-D-binding protein, kidney injury molecule- $I$, and interleukin- I 8}

These biomarkers have been subject of very recent investigations. Vitamin-D-binding protein (VDBP) is a low molecular weight protein that is filtered through the glomerulus as a 25-(OH) vitamin D 3/VDBP complex. In the normal kidney, VDBP is reabsorbed and catabolized by proximal tubular epithelial cells reducing urinary excretion to trace amounts. Acute tubular injury is expected to result in urinary VDBP loss. ${ }^{9}$ VDBP and kidney injury molecule-1 were identified as early and very sensitive markers of contrast-induced nephropathy, ${ }^{9}$ whereas interleukin-18 and kidney injury molecule-1 predicted early-stage AKI in burn patients. ${ }^{10}$

\section{Biomarkers of sepsis: a seven-league boots advance}

Sepsis is the most frequent cause of death in noncoronary ICUs. Despite better awareness and more efficient therapeutic support, the mortality of sepsis remains unacceptably high. In view of an often skewed clinical presentation, laboratory diagnosis is of utmost importance. During the past decade, a myriad of biomarkers for diagnosis and prognosis in septic patients has been proposed. However, most tests are either poorly sensitive or too complex and time consuming to be relevant for bedside use. An ideal biomarker has exquisite diagnostic sensitivity and specificity, high availability, is easy-to-use for decision making at the bedside, and costeffective. ${ }^{11} \mathrm{C}$-reactive protein (CRP) and procalcitonin (PCT) are the preferred markers of sepsis. Yet, many caveats regarding their use need consideration. CRP is produced in the liver and often fails to distinguish infection from inflammation in chronic liver disease. CRP also performs poorly in immunosuppressed patients (eg, patients receiving corticosteroids and transplant recipients). PCT has been promoted as a more infection-specific marker as it is generated by parafollicular thyroid cells and neuroendocrine cells in the lungs and intestine. ${ }^{12-14}$ PCT performs better than CRP, but many clinicians are reluctant to use it as a gold standard. A highly relevant issue is the behavior of CRP and PCT in patients undergoing CRRT. CRP is a $115 \mathrm{kDa}$ molecule formed by five monomers that are bound together by relatively fragile calcium bridges. ${ }^{16-18}$ Most CRP circulates in the blood as a $22-25 \mathrm{kDa}$ monomer. Theoretically, monomeric CRP is removed by CRRT as its molecular weight is below the cutoff of classical membranes (ie, $35 \mathrm{kDa}$ ). In addition, monomeric CRP is also removed by adsorption on the dialysis membrane. ${ }^{12-14} \mathrm{PCT}$ is a small $13 \mathrm{kDa}$ molecule and is only moderately removed by convection during CRRT. However, highly adsorptive membranes such as the AN69 (including the surface-treated type) and polymethylmethacrylate filters significantly boost PCT removal. The increasing application of highly adsorptive membranes for CRRT in most ICUs may significantly undermine the diagnostic and prognostic value of CRP and PCT as markers of infection. ${ }^{12-14}$

An upcoming marker could be hemoglobin subunit beta (HB $\beta)$, a component of hemoglobin. ${ }^{15}$ Hemoglobin is the main carrier of oxygen and carbon dioxide in cells of the erythroid lineage and ensures tissue oxygen delivery throughout the body. Blood HB $\beta$ levels in septic patients are significantly higher than those in healthy volunteers, and values in septic shock exceed those found in severe sepsis. HB $\beta$ levels apparently correlate with the degree of sepsisinduced endothelial cell dysfunction and thus may closely reflect sepsis severity. ${ }^{15}$

An area of particular clinical interest is the behavior of biomarkers dedicated to detect a specific pathology (such as NGAL for AKI) in sepsis. Indeed, increased NGAL levels are also observed in many septic patients. Moreover, as up to 
half of septic shock patients may develop AKI, NGAL measurement loses all specificity for detecting AKI , as it detect sepsis at the same time. ${ }^{16}$ Further research is needed to obtain biomarkers with sufficient diagnostic and prognostic specificity to discriminate between an ongoing septic process and an associated AKI. ${ }^{17}$ Research to interfere in this fascinating universe of interacting pathways is on the brink of a breakthrough. ${ }^{18}$

\section{Biomarkers of ARDS: looking beyond the lung?}

ARDS is a life-threatening complication of acute diseases such as sepsis, pneumonia, trauma, acute pancreatitis, aspiration of gastric contents, and near drowning. ARDS is characterized by diffuse alveolar injury, lung edema, neutrophil-derived inflammation, and surfactant dysfunction. Clinical manifestations are decreased lung compliance, severe hypoxemia, and bilateral pulmonary infiltrates. Sensitive biomarkers in plasma, tracheobronchial aspirate, or bronchoalveolar lavage fluid would surely improve severity staging and facilitate differentiation between mild, moderate, and severe ARDS. Also, changes in specific biomarker concentrations may quantitate evolving or receding lung injury and identify the activation of specific types of lung cells (eg, epithelial vs endothelial cells, neutrophils vs macrophages). This might represent an immense step forward in evaluating the patient's evolution under current or experimental therapy. ${ }^{19}$ Among recently proposed markers, endocan aka endothelial cell-specific molecule-1 has considerable potential. This soluble $50 \mathrm{kDa}$ dermatan sulfate proteoglycan is secreted by both pulmonary and kidney vascular endothelial cells. ${ }^{20}$ Plasma levels are dramatically increased in pneumonia patients with ARDS but not in subjects without ARDS. Endocan is also a promising biomarker to predict disease severity and mortality in patients with ARDS. ${ }^{21}$ Angiogenic and inflammatory factors in ARDS patients may be relevant for AKI since they can mediate interorgan "cross talk" expanding into simultaneous occurrence of ARDS and AKI. For example, patients with influenza A/H1N1-induced ARDS who develop AKI excessively produce monocyte chemotactic protein 1 , vascular endothelial growth factor, and interferon gammainduced protein $10 .{ }^{22}$ This explosive cocktail likely contributes to kidney injury and is associated with a higher risk of death. ${ }^{23}$

\section{Conclusion}

Through the years, biomarker research has advanced by giant leaps. Sensitivity of biomarkers has improved consid- erably but specificity remains poor, in particular when two "marker-sensitive" conditions overlap in one patient (eg, sepsis and AKI; AKI and ARDS). Biomarkers are already important adjuvant tools for refining and optimizing diagnosis, treatment, and prognosis of AKI and infection. In postoperative and critically ill patients who typically present varying degrees of inflammation, infection, and concomitant (sub)acute organ dysfunction or failure, biomarkers are "hot" and promising but not yet ready for prime time. ${ }^{1}$

\section{Authors' contributions}

All authors contributed toward data analysis, drafting and critically revising the paper and agree to be accountable for all aspects of the work .

\section{Disclosure}

The authors report no conflicts of interest in this work.

\section{References}

1. Honore PM, Jacobs R, Joannes-Boyau O, et al. Biomarkers for early diagnosis of AKI in the ICU: ready for prime time use at the bedside? Ann Intensive Care. 2012;2(1):24.

2. Haase M, Devarajan P, Haase-Fielitz A, et al. The outcome of neutrophil gelatinase-associated lipocalin-positive subclinical acute kidney injury: a multicenter pooled analysis of prospective studies. $\mathrm{JAm}$ Coll Cardiol. $2011 ; 57(1): 1752-1761$

3. Haase M, Kellum JA, Ronco C. Subclinical AKI--an emerging syndrome with important consequences. Nat Rev Nephrol. 2012;8(12):735-739.

4. Ronco C, Kellum JA, Haase M. Subclinical AKI is still AKI. Crit Care. 2012;16(3):313

5. Ronco C, Stacul F, McCullough PA. Subclinical acute kidney injury (AKI) due to iodine-based contrast media. Eur Radiol. 2013;23(2) 319-323.

6. Kashani K, Al-Khafaji A, Ardiles T, et al. Discovery and validation of cell cycle arrest biomarkers in human acute kidney injury. Crit Care. 2013;17(1):R25

7. Hoste EA, McCullough PA, Kashani K, et al; Sapphire Investigators. Derivation and validation of cutoffs for clinical use of cell cycle arrest biomarkers. Nephrol Dial Transplant. 2014;29(11):2054-2061.

8. Gaygısız Ü, Aydoğdu M, Badoğlu M, Boyacı N, Güllü Z, Gürsel G. Can admission serum cystatin $\mathrm{C}$ level be an early marker subclinical acute kidney injury in critical care patients? Scand J Clin Lab Invest. 2016;76(2):143-150.

9. Chaykovska L, Heunisch F, von Einem G, et al . Urinary vitamin D binding protein and KIM-1 are potent new biomarkers of major adverse renal events in patients undergoing coronary angiography. PLoS One. 2016;11(1):e0145723.

10. Ren H, Zhou X, Dai D, et al. Assessment of urinary kidney injury molecule-1 and interleukin-18 in the early post-burn period to predic acute kidney injury for various degrees of burn injury. BMC Nephrol. 2015;16:142.

11. Prucha M, Bellingan G, Zazula R. Sepsis biomarkers. Clin Chim Acta 2015;440:97-103

12. Honoré PM, Jacobs R, De Waele E, Van Gorp V, Spapen HD. Biomarkers of inflammation during continuous renal replacement therapy: sensors, players, or targets? A reply to the letter by Villa et al. Blood Purif. 2014;38(2):102-103.

13. Honore PM, Jacobs R, De Waele E, Spapen HD. Biomarkers to detect sepsis: a "burning" issue but still a long way to go*. Crit Care Med. 2014; 42(9):2137-2138 
14. Honore PM, Jacobs R, Hendrickx I, De Waele E, Van Gorp V, Spapen HD. "Biomarking" infection during continuous renal replacement therapy: still relevant? Crit Care. 2015;19:232.

15. Yoo H, Ku SK, Kim SW, Bae JS. Early diagnosis of sepsis using serum hemoglobin subunit beta. Inflammation. 2015;38(1):394-399.

16. Hjortrup PB, Haase N, Treschow F, Møller MH, Perner A. Predictive value of NGAL for use of renal replacement therapy in patients with severe sepsis. Acta Anaesthesiol Scand. 2015;59(1):25-34.

17. Ronco C, Legrand M, Goldstein SL, et al. Neutrophil gelatinaseassociated lipocalin: ready for routine clinical use? An international perspective. Blood Purif. 2014;37(4):271-285.

18. Honore PM, Nguyen HB, Gong M, et al; Sapphire and Topaz Investigators. Urinary tissue inhibitor of metalloproteinase-2 and insulin-like growth factor-binding protein 7 for risk stratification of acute kidney injury in patients with sepsis. Crit Care Med. 2016 Jun 28. [Epub ahead of print].
19. Mokra D, Kosutova P. Biomarkers in acute lung injury. Respir Physiol Neurobiol. 2015;209:52-58.

20. Aschner Y, Zemans RL, Yamashita CM, Downey GP. Matrix metalloproteinases and protein tyrosine kinases: potential novel targets in acute lung injury and ARDS. Chest. 2014;146(4):1081-1091.

21. Tang L, Zhao Y, Wang D, et al. Endocan levels in peripheral blood predict outcomes of acute respiratory distress syndrome. Mediators Inflamm. 2014;2014:625180.

22. Bautista E, Arcos M, Jimenez-Alvarez L, et al. Angiogenic and inflammatory markers in acute respiratory distress syndrome and renal injury associated to A/H1N1 virus infection. Exp Mol Pathol. 2013;94(3): 486-492.

23. Kute VB, Godara SM, Goplani KR, et al. High mortality in critically ill patients infected with 2009 pandemic influenza A (H1N1) with pneumonia and acute kidney injury. Saudi J Kidney Dis Transpl. 2011;22:83-89.
The International Journal of Nephrology and Renovascular Disease is an international, peer-reviewed open access journal focusing on the pathophysiology of the kidney and vascular supply. Epidemiology, screening, diagnosis, and treatment interventions are covered as well as basic science, biochemical and immunological studies. The manuscript management system is completely online and includes a very quick and fair peer-review system, which is all easy to use. Visit http://www. dovepress.com/testimonials.php to read real quotes from published authors.

Submit your manuscript here: https://www.dovepress.com/international-journal-of-nephrology-and-renovascular-disease-journal 